Manuelle Medizin 2013 · 51:168-168 DOI 10.1007/s00337-013-1016-x Online publiziert: 10. Februar 2013

(c) Springer-Verlag Berlin Heidelberg 2013

\title{
Beobachtung bestätigt
}

\section{Zum Beitrag \\ Göhmann U, Sacher R, Kayser C (2012) \\ Hüftsonographie: Einmal gut, immer gut? \\ Manuelle Med 50:515-518}

\section{Leserbrief}

\section{Seifert, Chemnitz}

Sehr geehrte Kollegen Göhmann, Sacher und Kayser,

mit Freude habe ich Ihren Artikel gelesen und kann Ihre Beobachtung nur bestätigen. In unserer Praxis beobachteten wir mehrfach Säuglinge, bei denen wir im Beobachtungszeitraum von einigen Monaten Hüftdysplasien feststellten, obgleich die Eingangsuntersuchung sonographisch ideal war. Zweifel an der Sonographietechnik und/oder -auswertung bestätigten sich weder in den eigenen Befunden noch in den Befunden der vorbehandelnden Kollegen. Alle Säuglinge waren wegen „Schräglagen“ in unserer Behandlung mit unterschiedlichen Befunden.

Über den Zusammenhang kann man spekulieren. Der Einfluss der Muskulatur auf die Entwicklung des Hüftgelenks ist uns ja allen beim heranwachsenden Kind bekannt, z. B. ist die Entwicklung einer Coxa valga bei Adduktorenspastik die Regel. Auf diesem Wege ist auch die Veränderung der Säuglingshüfte über eine veränderte Muskelspannung denkbar, ob über den Weg der myofaszialen „Verkettung“ oder die engen direkten Zusammenhänge von Iliosakral- und Hüftgelenk. Dabei gehe ich nicht auf die Frage der Priorität von Ursache und Wirkung ein. Es gibt durchaus Lageasymmetrien bei Säuglingen ohne Störung der Kopfgelenke.
Ihre Schlussfolgerung, Säuglinge trotz guter Anfangsbefunde weiter sonographisch zu kontrollieren, kann ich nur unterstützen.

Mit freundlichen Grüßen

\section{Korrespondenzadresse}

\section{Dr. I. Seifert}

Praxis für Orthopädie

Drosselweg 4, 09130 Chemnitz

dr-irmgard-seifert@freenet.de 